\section{Overlap between frontotemporal dementia and Alzheimer's disease: case report}

\author{
Maria Elena Di Battista, \\ Maurizio Gallucci
}

Cognitive Impairment Center, Local Health Authority n. 2 Marca Trevigiana, Treviso, Italy

\begin{abstract}
The frontal variant of Alzheimer's disease (fv-AD) has been described in patients with prominent behavioral or executive dysfunctions. Subsequently, the spectrum of frontal variant Alzheimer's disease has been enlarged to comprise patients with early personality and behavioral changes including disinhibition, apathy or compulsiveness.

We describe the case of a patient with a history of memory loss and behavioral changes. The neuropsychological profile overlapped with the presence of behavioral disorders such as marked apathy, disinhibition, hostile behavior, agitation, irritability and hyperorality.
\end{abstract}

The results of the neuropsychological examination leaned towards a diagnosis of frontotemporal circuit functional impairment, however, the 18-FDG PET study demonstrated a moderate-to-severe impairment in the bilateral parietal regions.

On the basis of the neuropsychological profile and 18-FDG PET imaging, a diagnosis of a probable fv-AD was made, the patient started oral rivastigmine $3 \mathrm{mg} /$ daily and subsequent assessments showed only modest worsening in the cognitive profile and a moderate improvement in behavioral symptoms.

\section{Case Report}

We report on the case of a 56-year-old man with a history of behavioral disorders and memory loss. In the report, we investigate the differential diagnosis between the behavioral variant of frontotemporal dementia (bv-FTD) and the frontal variant of Alzheimer's disease.

A 56-year-old male patient was admitted to our Centre for Dementia and Cognitive Disorders in September 2015. No relevant diseases were present in the clinical history of the patient and no family history of neurodegenerative or psychiatric diseases was reported. Two years before our visit the patient began to experience memory loss, spatial disorientation, behavioral changes, irritability and severe apathy. Disorders of social behavior and impaired executive functions were prominent and the patient lost his job as a truck driver. Before admittance to our Centre the patient underwent a psychiatric assessment and a neuropsychological examination. As a result, therapy with haloperidol $1 \mathrm{mg} /$ die was prescribed without, however, any significant clinical improvement.

During the medical examination at our Center the patient was aggressive towards his wife, demonstrating marked irritability often with an altered tone of voice, alongside sudden outbursts of anger during which he would suddenly get out of his chair and leave the room.

The neurological examination was normal. Blood examination revealed a moderate hyperhomocysteinemia $(17.9 \mu \mathrm{mol} / \mathrm{L})$. The patient then had a magnetic resonance imaging (MRI) scan (Figure 1) that showed no signal alterations in brain tissue and no atrophy in the cortical or subcortical structures.

During the neuropsychological evaluation the patient proved lucid, conscious only of his memory disorders. Furthermore, his behavior was not completely suitable to the context in which he found himself: he tried to collude with the examiner by being overly friendly.

His spontaneous speech was fluent and well organized from a syntactic grammatical point of view. His level of oral understanding of spoken language was maintained. A frequent tendency to ramble and to repeat content already expressed was observed. He looked well oriented.

The neuropsychological examination showed deficits in memory with performances on Rey auditory verbal learning test under the cut-off values (RAVLT-IR $=24.2$ with normal values $>28.53$, and RAVLT-DR $=0.6$, with normal value $>4.69$ ); the short story test also showed a memory deficit.

The frontal assessment battery (FAB) showed pathological executive functions. The constructional praxis test was also abnormal with design copying under the cut-off value.

Tests measuring attention, phonemic and sematic verbal fluency were normal.

Overall neuropsychological tests showed cognitive deficits in the amnesic domain, in executive functions and constructional apraxia.

The above described neuropsychological profile overlapped with the presence of behavioral disorders such as marked apathy, disinhibition, hostile behavior, agitation, irritability and hyperorality as evidenced by the neuropsychiatric inventory scale (NPI).
Correspondence: Maria Elena Di Battista, Cognitive Impairment Center, Local Health Authority n. 2 Marca Trevigiana, Borgo

Cavalli 42, 31100 Treviso, Italy.

Tel.: +39.0422.323395 - Fax:

+39.0422 .323329 .

E-mail:

mariaelena.dibattista@aulss2.veneto.it

Key words: Frontal variant of Alzheimer's disease; early-onset Alzheimer's disease; Alzheimer's behavioral symptoms.

Contributions: the authors contributed equally.

Conflict of interest: the authors declare no conflict of interest.

Received for publication: 23 June 2017 Accepted for publication: 11 July 2017.

This work is licensed under a Creative Commons Attribution-NonCommercial 4.0 International License (CC BY-NC 4.0).

(C) Copyright M.E. Di Battista and M. Gallucci, 2017

Licensee PAGEPress, Italy

Geriatric Care 2017; 3:6866

doi:10.4081/gc.2017.6866

Taken together, the results of the neuropsychological examination leaned towards a diagnosis of frontotemporal circuit functional impairment. According to current clinical criteria, a diagnosis of possible bv-FTB could be made in agreement with the neuropsychological and psychiatric profile that we found. ${ }^{1}$

However, given that the typical imaging pattern of frontotemporal brain atrophy was absent and so as to gain a better definition of the case, the patient underwent further investigation.

The 18-FDG PET study demonstrated a moderate-to-severe impairment in the bilateral parietal regions, with slight prevalence on the right; a mild deficit in glucose metabolic activity was also found in the posterior cingulate and in the frontal and temporal bilateral lobes (Figures 2 and 3 ).

A cerebrospinal fluid (CSF) sample to measure biomarkers was obtained. The CSF profile was fairly normal, with amyloid $\beta$ 42 of $605 \mathrm{ng} / \mathrm{L}$, total Tau $503 \mathrm{ng} / \mathrm{L}$, p-Tau $67 \mathrm{ng} / \mathrm{L}$ and $\mathrm{A} \beta 42 / \mathrm{p}$-Tau ratio 9.0.

On the basis of the neuropsychological profile and 18-FDG PET imaging, a diagnosis of a frontal probable variant of Alzheimer's disease (fv-AD) was made, notwithstanding the CSF profile which was only weakly positive with respect to the Tau and the structural neuroimaging findings on MRI. 
In January 2016 the patient started oral rivastigmine $3 \mathrm{mg} /$ daily and assessments performed in March and November 2016 and May 2017 showed only modest worsening in the cognitive profile and a moderate improvement in behavioral symptoms.

\section{Discussion}

The fv-AD has been described in patients with prominent behavioral or executive dysfunctions produced by the characteristic Alzheimer's disease pathology.

The term of frontal variant of Alzheimer's disease was formerly conceptualized by Johnson et al. ${ }^{2}$ in patients with early and predominant executive dysfunction bearing the classic hallmarks of AD pathology, namely amyloid plaque and neurofibrillary tangle. Subsequently, the spectrum of frontal variant Alzheimer's disease has been enlarged to comprise patients with early personality and behavioral changes including disinhibition, apathy or compulsiveness.

On a general level, it is worth noting that the clinical presentation of frontal variant Alzheimer's disease may imitate that of behavioral variant frontotemporal dementia (bv-FTD). The clinical overlap between these two conditions is highlighted by the observation that a proportion ranging from 10 to $40 \%$ of patients diagnosed with behavioral variant FTD are found to have AD-like pathology on post-mortem examination ${ }^{3,4}$ and positive amyloid PET imaging. ${ }^{5,6}$

We suggest that the distinguishing findings of our case, namely the lack of temporal atrophy on MRI neuroimaging and the not overwhelming CSF profile may possibly be related to the relative youth of our patient.

Of note, late-onset (LOAD) and earlyonset (EOAD) Alzheimer's disease affect a variety of neural systems likely reflecting partially different clinical and pathological entities. In these terms, several lines of evidence demonstrate that the classic hallmark of LOAD, namely medial temporal lobe atrophy, is not frequently found in EOAD patients. ${ }^{7}$ Furthermore, patients with nontemporal AD subtype have a distinct clinical phenotype regarding their cognitive deficit profile with prominent dysfunctions in attention, visuospatial, and frontal-executive domains when compared to the classic AD phenotype. Imaging studies on cortical thickness support the notion that younger $\mathrm{AD}$ patients with non-hippocampal neuropsychological profiles present a different pattern of cortical involution involving parietal lobes rather than temporal regions. ${ }^{8}$

Although neuropathological evidence indicates that EOAD and LOAD have the same features and represent a continuum of the same pathological processes, the differences between EOAD and LOAD are reported also in metabolic studies. Indeed,
EOAD patients present a singular $18 \mathrm{~F}-\mathrm{FDG}$ PET imaging pattern with hypometabolism of the mesial temporal and parietal lobes (i.e., precuneus, lateral parietal and occipi-

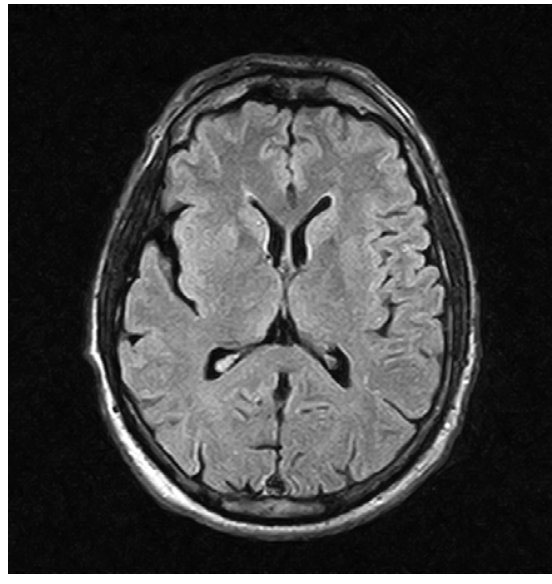

Figure 1. T2 FLAIR MRI. No signal alterations in brain tissue and no atrophy in the cortical or subcortical structures.

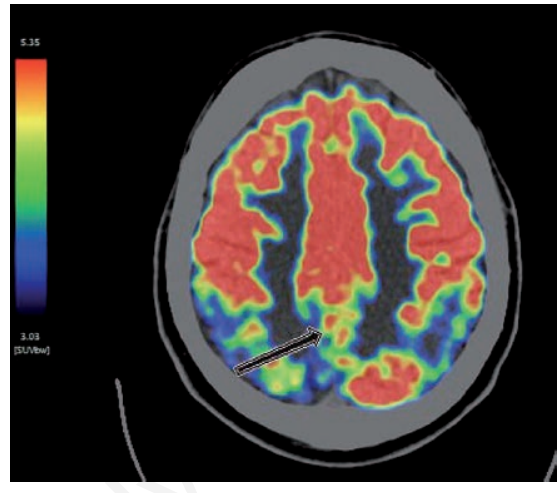

Figure 2. Axial 18-FDG PET scan. Moderate-to-severe impairment in the bilateral parietal regions, with slight prevalence on the right; a mild deficit in glucose metabolic activity was also found in the posterior cingulate (arrow).

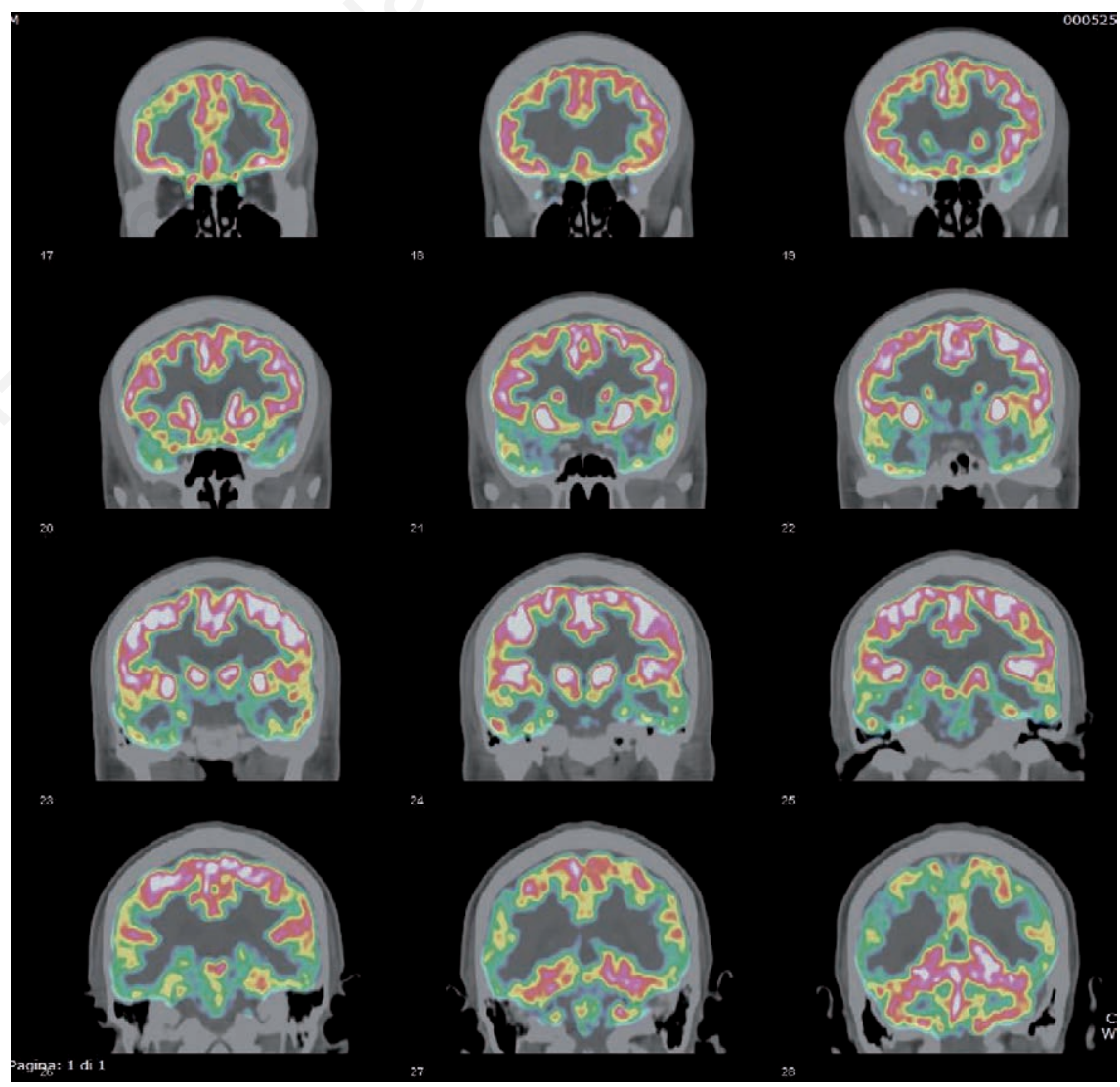

Figure 3. Coronal 18-FDG PET scan. Moderate-to-severe impairment in the bilateral parietal regions, with slight prevalence on the right; a mild deficit was also found in the frontal and temporal bilateral lobes. 
tal brain regions) and relative sparing of hippocampal structures with respect to LOAD. In addition, EOAD patients tend to have a more aggressive rate of progression with a shorter duration of the disease than LOAD patients.

FDG-PET also demonstrate a different profile in early $v s$ late-onset $\mathrm{AD}$ with respect to the neuropsychological profile. ${ }^{9}$ In particular, EOAD patients that display worse executive functions, present greater hypometabolism in the parietal regions, whereas LOAD patients with worse memory performance have greater hypometabolism in inferior frontotemporal regions.

Finally, there is evidence suggesting that CSF in AD-like conditions show similar profiles, confirming the usefulness of CSF testing in terms of differential diagnosis. ${ }^{10}$ In particular, the core diagnostic proteins for $\mathrm{AD}$, namely amyloid- $\beta 42$, total Tau and $\mathrm{p}$ Tau, present poor predictive power for other conditions such as FTD. According to evidence from a variety of sources, the sensitivity of amyloid 42 is estimated at about $67 \%$, which means that one third of the patients would not be identified. ${ }^{11}$

Furthermore, there is consistent variability in the methods used to collect and analyze CSF, leading to partially conflicting results. ${ }^{12}$

\section{Conclusions}

The clinical presentation of frontal variant Alzheimer's disease may imitate that of behavioral variant frontotemporal dementia (bv-FTD).

It is worth considering that bv-FTD and frontal variant Alzheimer disease (fv-AD) may present an important clinical overlap. In such conditions, no one single biomarker is diagnostic and interpretation of the results should always be made in the context of the single case in analysis. ${ }^{12}$

\section{References}

1. Rascovsky K, Hodges JR, Knopman D, et al. Sensitivity of revised diagnostic criteria for the behavioural variant of frontotemporal dementia Katya. Brain 2011;134;2456-77.

2. Johnson JK, Head E, Kim R, et al. Clinical and pathological evidence for a frontal variant of Alzheimer disease. Arch Neurol 1999;56:1233-9.

3. Varma AR, Snowden JS, Lloyd JJ, et al. Evaluation of the NINCDS-ADRDA criteria in the differentiation of Alzheimer's disease and frontotemporal dementia. J Neurol Neurosurg Psychiatry 1999;66: 184-8.

4. Alladi S, Xuereb J, Bak T, et al. Focal cortical presentations of Alzheimer's disease. Brain 2007;130:2636-45.

5. Laforce R Jr, Rabinovici GD. Amyloid imaging in the differential diagnosis of dementia: review and potential clinical applications. Alzheimers Res Ther 2011; 10:3-31.
6. Morbelli S, Perneczky R, Drzezga A, et al. Metabolic networks underlying cognitive reserve in prodromal Alzheimer disease: a European Alzheimer disease consortium project. J Nucl Med 2013;54: 894-902.

7. Cavedo E, Pievani M, Boccardi M, et al. Medial temporal atrophy in early and late-onset alzheimer's disease. Neurobiol Aging 2014;35:2004-12.

8. Noh Y, Jeon S, Lee JM, et al. Anatomical heterogeneity of Alzheimer disease: based on cortical thickness on MRIs. Neurology 2014;18:1936-44.

9. Kaiser NC, Melrose RJ, Liu C, et al. Neuropsychological and neuroimaging markers in early versus late-onset Alzheimer's disease. Am J Alzheimers Dis Other Demen 2012;27:520-9.

10. Rostgaard N, Waldemar G, Nielsen JE, Simonsen AH. Cerebrospinal fluid biomarkers in familial forms of Alzheimer's disease and frontotemporal dementia. Dement Geriatr Cogn Disord 2015;40: 54-62.

11. Stomrud E, Minthon L, Zetterberg H, et al. Longitudinal cerebrospinal fluid biomarker measurements in preclinical sporadic Alzheimer's disease: a prospective 9-year study. Alzheimer's Dementia Diagn Assess Dis Monitor 2015;1:403-11.

12. Ahmed RM, Paterson RW, Warren JD, et al. Biomarkers in dementia: clinical utility and new directions. J Neurol Neurosurg Psychiatry 2014;85:1426-34. 